\title{
Ecological speciation in sympatric palms: 4. Demographic analyses support speciation of Howea in the face of high gene flow
}

Papadopulos, Alexander S. T.; Igea, Javier; Smith, Thomas P.; Hutton, Ian; Baker, William J.; Butlin, Roger K.; Savolainen, Vincent

\section{Evolution}

DOI:

10.1111/evo.13813

10.1111/evo.13813

Published: 01/09/2019

Peer reviewed version

Cyswllt i'r cyhoeddiad / Link to publication

Dyfyniad o'r fersiwn a gyhoeddwyd / Citation for published version (APA):

Papadopulos, A. S. T., Igea, J., Smith, T. P., Hutton, I., Baker, W. J., Butlin, R. K., \& Savolainen, V. (2019). Ecological speciation in sympatric palms: 4. Demographic analyses support speciation of Howea in the face of high gene flow. Evolution, 73(9), 1996-2002.

https://doi.org/10.1111/evo.13813, https://doi.org/10.1111/evo.13813

\footnotetext{
Hawliau Cyffredinol / General rights

Copyright and moral rights for the publications made accessible in the public portal are retained by the authors and/or other copyright owners and it is a condition of accessing publications that users recognise and abide by the legal requirements associated with these rights. study or research.

dow public portal for the purpose of private

- You may not further distribute the material or use it for any profit-making activity or commercial gain

- You may freely distribute the URL identifying the publication in the public portal ?
}

Take down policy

If you believe that this document breaches copyright please contact us providing details, and we will remove access to the work immediately and investigate your claim. 


\section{BRIEF COMMUNICATION}

\section{Ecological speciation in sympatric palms: 4. Demographic analyses support speciation of}

Howea in the face of high gene flow

Alexander S.T. Papadopulos ${ }^{1,2}$, Javier Igea ${ }^{1,3}$, Thomas P. Smith ${ }^{1}$, Ian Hutton ${ }^{4}$, William J. Baker ${ }^{5}$, Roger K. Butlin ${ }^{6,7}$ and Vincent Savolainen ${ }^{1,5, *}$

${ }^{1}$ Department of Life Sciences, Silwood Park Campus, Imperial College London, Buckhurst Road, Ascot, SL5 7PY, UK.

${ }^{2}$ Molecular Ecology and Fisheries Genetics Laboratory, Environment Centre Wales, School of Biological Sciences, Bangor University, Bangor, LL57 2UW, UK.

${ }^{3}$ Department of Plant Sciences, University of Cambridge, Cambridge, CB2 3EA, UK.

${ }^{4}$ Lord Howe Island Museum, Lord Howe Island, New South Wales, Australia.

${ }^{5}$ Royal Botanic Gardens, Kew, Richmond, TW9 3AB, UK.

${ }^{6}$ Department of Animal and Plant Sciences, University of Sheffield, Sheffield, S10 2TN, UK.

${ }^{7}$ Department of Marine Sciences, University of Gothenburg, Gothenburg, SE-405 30, Sweden

*Correspondence: email v.savolainen@imperial.ac.uk

Running title (40 characters) Gene flow and speciation in Howea palms

Keywords Coalescence, sympatry, speciation, ddRAD

\section{AUTHOR CONTRIBUTIONS}

VS designed the research with contributions from ASTP, JI, and RKB. ASTP and TPS collected data. ASTP and JI analysed the data. IH and WB contributed to field collections. ASTP and VS wrote the manuscript. All authors commented on the manuscript.

\section{ACKNOWLEDGMENTS}

We thank the Lord Howe Island Board and the New South Wales National Park and Wildlife Services for granting research permits, Hank and Sue Bower, Christo Haselden, Peter Weston, Larry Wilson for their help on LHI, Laszlo Csiba and Helen Hipperson for help in the lab, Matt Hahn for comments, and the European Research Council, NERC and the Leverhulme Trust for funding.

\section{DATA ACCESSIBILITY}

The sequence data are available at the Sequence Reads Archive under accession numbers PRJNA386480 and SRP063985. 
Ecological speciation in sympatric palms: 4. Demographic analyses support speciation of Howea in the face of high gene flow

\begin{abstract}
The idea that populations must be geographically isolated (allopatric) to evolve into separate species has persisted for a long time. It is now clear that new species can also diverge despite ongoing genetic exchange, but few accepted cases of speciation in sympatry have held up when scrutinised using modern approaches. Here, we examined evidence for speciation of the Howea palms of Lord Howe Island, Australia, in light of new genomic data. We used coalescence-based demographic models combined with double digest restriction-site associated DNA sequencing of multiple individuals and provide support for previous claims by Savolainen et al. (Nature 441: 210 $213,2006)$ that speciation in Howea did occur in the face of gene flow.
\end{abstract}

\title{
Introduction
}

Sympatric speciation has re-emerged as a controversial topic, with recent analyses of genome-wide data casting doubt on some of the most best-known examples, such as cichlid fish in Cameroonian crater lakes (Martin et al. 2015). Due to a lack of confidence in ascertaining whether speciation has taken place in the face of gene flow, our understanding of the genomic underpinning of such processes has also remained piecemeal (Renaut et al. 2013; Cruickshank \& Hahn 2014). Here, we examined a case of speciation in Howea palms, a genus that comprises only two species, both endemic to the subtropical Lord Howe Island (LHI; Savolainen et al. 2006). The island is isolated (600 km from mainland Australia) and minute $\left(<16 \mathrm{~km}^{2}\right)$. Furthermore, modelling of the geological history and sizes of LHI and nearby Ball's Pyramid rock showed that, for any pair of endemic sister species that have diverged within the lifetime of the island, an allopatric phase in their divergence is unlikely (Papadopulos et al. 2011). This was critical in promoting Howea as a prime example of sympatric speciation under a biogeographic definition (Savolainen et al. 2006; Mallet et al. 2009; Coyne 2011; Papadopulos et al. 2011). Marked flowering time differences between the species indicate that prezygotic isolation is now strong and current levels of gene flow are likely to be low (Savolainen et al. 2006; Babik et al. 2009; Dunning et al. 2016; Hipperson et al. 2016). Indirect evidence of post-zygotic isolation due to selection against juvenile hybrids supports the hypothesis that divergent selection has influenced the speciation process (Hipperson et al. 2016). Given that the distributions of Howea palms overlap extensively and that they are wind pollinated, Savolainen $e t$ al. (2006) argued that speciation is likely to have occurred in the face of high gene flow, which may have reduced quickly as divergence progressed (Savolainen et al. 2006; Babik et al. 2009; 
Papadopulos et al. 2011, 2013b, 2014). However, previous efforts were based on a limited number of markers (two gene sequences and amplified fragment length polymorphism; Savolainen et al. 2006, Babik et al. 2009), which leaves room for doubt about the precise timing of divergence and did not allow for the quantification of the extent of the gene exchange between $H$. forsteriana and $H$. belmoreana. Here, we characterise the demographic history of the Howea palms with a genomewide dataset to evaluate (i) whether genomic data support speciation with gene flow and (ii) how genetic exchange progressed during speciation in sympatry.

\section{Material and Methods}

\section{DNA EXTRACTION AND DOUBLE DIGEST RAD-SEQUENCING}

Leaf tissue was collected and preserved in silica gel from $42 \mathrm{H}$. belmoreana and $54 \mathrm{H}$. forsteriana individuals sampled at Far Flats on LHI where both species co-occur. Genomic DNA was extracted from leaf tissue using DNeasy Plant Mini kits (Qiagen). Agarose gel electrophoresis was used to assess the quality of each DNA extract and DNA quantification was performed with a Qubit 2.0 fluorometer (Life technologies). DNA samples were then processed following a modified version of the double digest RADseq protocol of Peterson et al. (2012). Digestion of the DNA template of each sample (250-1000ng) was performed by combining the sample with $0.1 \mu 1$ EcoRI-HF (10 U; NEB), $1 \mu 1 \mathrm{MspI}$ (10U; NEB), $5 \mu \mathrm{l}$ NEB buffer 4, and nuclease-free water to a total volume of $50 \mu 1$. Digestion reactions were incubated at $37^{\circ} \mathrm{C}$ for 3 hours. Digests were cleaned using the Agencourt AMPure XP bead clean up (Beckman Coulter) and quantified using a Qubit 2.0 fluorometer. Ligation of 6 base pairs (bp) - barcoded P1 (EcoRI overhang) and P2 (MspI) adaptors was performed in individual reactions composed of ca. $400 \mathrm{ng}$ of the digested DNA product, $2 \mu \mathrm{l}$ of each adaptor (4 nM), $2 \mu 1$ T4 DNA ligase (4000 U; NEB), $4 \mu 1$ 10x Ligase buffer (NEB) and nuclease-free water to a total of volume $40 \mu$ l. Ligation reactions were incubated at $23^{\circ} \mathrm{C}$ for 30 min, followed by $65^{\circ} \mathrm{C}$ for $10 \mathrm{~min}$, and then cooled to $18^{\circ} \mathrm{C}$ at a rate of $2^{\circ} \mathrm{C} / 90$ seconds. Samples were pooled into batches of 12 containing compatible sets of barcodes, and cleaned using Agencourt AMPure XP beads. Each pool was size-selected between 344-408 bp using a Pippin Prep electrophoresis system (Sage Science). Each size-selected pool was PCR amplified in 6-8 reactions each composed of $6 \mu 1$ of DNA template, $2 \mu 1$ of each ddRAD primer, $0.2 \mu 1$ Phusion Taq polymerase (NEB), $0.4 \mu 1$ dNTPs (10 mM; NEB), $4 \mu 1$ 5x Phusion HF buffer (NEB) and $5.4 \mu 1$ nuclease-free water. PCR reactions were run on a Veriti 96-Well Fast Thermal Cycler (Applied Biosystems) at $98^{\circ} \mathrm{C}$ for $30 \mathrm{~s}, 12$ cycles of $98^{\circ} \mathrm{C}$ for $15 \mathrm{~s}, 65^{\circ} \mathrm{C}$ for $30 \mathrm{~s}, 72^{\circ} \mathrm{C}$ for $30 \mathrm{~s}$, and final $72^{\circ} \mathrm{C}$ for $7 \mathrm{~min}$. PCR reactions for each pool were combined and cleaned using Agencourt AMPure XP beads. Each cleaned pool was diluted to $4 \mathrm{nM}$ and four pools with compatible barcodes were 
combined to produce libraries of 48 uniquely barcoded samples. Two ddRAD sequence libraries were prepared in this way. All libraries were sequenced to $100 \mathrm{bp}$, paired-end on an Illumina Hiseq 2500 (one lane per library) at the MRC Clinical Sciences Centre, Imperial College London. This generated an average of 6,186,469 reads per sample. Genotyping of ddRAD data was performed using the STACKS pipeline, building upon the catalogue generated in Papadopulos et al. (2019b). To expand the catalogue to encompass haplotypes present in both Howea species, samples were assembled into loci using USTACKS (-m20, -M3) and these stacks were merged into the existing catalogue allowing 3 mismatches between loci in different individuals. To genotype individuals, loci were assembled with lower coverage in USTACKS (-m5, -M3) and these stacks were mapped to the catalogue loci.

\section{DEMOGRAPHIC MODELLING}

For the demographic analysis, the Far Flats individuals were genotyped at 4,581 loci (23,109 SNPs) with fewer than 20 missing individuals (minor allele frequency $=0.05$ ). To account for missing data, we projected the number of individuals down to 36/54 $\mathrm{H}$. forsteriana and 23/42 $\mathrm{H}$. belmoreana and calculated the joint folded site-frequency-spectrum using $\delta a \delta i$ (Gutenkunst et al. 2009). We then inferred the demographic history of Howea from the site-frequency-spectrum using two methods: (i) the composite-likelihood framework implemented by fastsimcoal2 (Excoffier et al. 2013) and (ii) the diffusion approximation approach implemented in a modified version of $\delta a \delta i$ (Gutenkunst et al. 2009; Tine et al. 2014). This modified version of $\delta a \delta i$ accounts for variation in the rate of gene flow across the genome by dividing the genome into two types of loci (in $\mathrm{P}$ and 1-P proportions) with potentially different migration rates.

For fastsimcoal2, we estimated parameters 60 times for each of 10 demographic models to determine the combination of parameters with the highest likelihood. These models are shown on Figure 1a and assume either no population growth, or population growth: model 1 - speciation without gene flow; model 2 - speciation with recent gene flow following secondary contact; model 3 - speciation with initial gene flow; model 4 - speciation with constant gene flow; and model 5 speciation with two distinct periods of migration where gene flow may vary. Model fit was assessed using the Akaike Information Criterion (AIC). Non-parametric bootstrapping (100 simulated datasets under the best model with 10 rounds of parameter estimation for each simulation) was used to estimate $95 \%$ confidence intervals for each parameter for the best model.

For $\delta a \delta i$, we compared the fit of the same 10 models as above plus another eight, that is, models 2-5 with and without population growth, but also including heterogeneous rates of migration across the 
genome. We ran two rounds of simulated annealing (one hot and one cold) followed by a final round of Broyden-Fletcher-Goldfarb-Shanno optimisation. For each of the 18 models, we performed a minimum of 30 runs to ensure thorough estimation of the maximum likelihood and used AIC to perform model selection. For the best fitting model, we then ran 30 bootstrap replicates (ensuring that each replicate had at least 10 runs) using the built-in $\delta a \delta i$ procedure to get confidence intervals around the parameter estimates.

To calibrate the demographic models in fastsimcoal 2 and $\delta a \delta i$, we estimated the substitution rate in Howea. We first built a phylogenetic tree using genome wide data for all available palm species. Transcriptome assemblies were obtained for H. forsteriana (Dunning et al 2016), Elaeis guineensis and E. oleifera (African and American oil palms; Singh et al. 2013). Short read transcript data for Phoenix dactilyfera (date palm; Al-Mssallem et al. 2013) were obtained from the Short Read Archive (Accession Number SRR341952) and a transcriptome assembly was produced using Trinity (Grabherr et al. 2011) with default parameters and min_kmer_cov set to 2. TransDecoder was used to predict open reading frames (ORF) with a minimum length of 100 amino acids, and the longest ORF was selected. Reciprocal blast hits for all four palm species were established by collating reciprocal best blast results from a pair of species with the remaining two species; this rendered an initial set of orthologous alignments for the four species. An $M$-coffee (Wallace et al. 2006) pipeline was used to score these alignments. The nucleotide sequences were translated into protein using t-coffee, then the protein sequences were aligned with MAFFT (Katoh \& Toh 2008), Muscle (Edgar 2004), t-coffee (Notredame et al. 2000) and k-align (Lassmann \& Sonnhammer 2005) and translated back to DNA. Low quality positions with scores lower than 8 were trimmed. Protein alignments were used to guide gap placement in the nucleotide alignments. Finally, maxalign was used to check for any misaligned sequences in the alignments (Gouveia-Oliveira et al. 2007). Only alignments where all the orthologous sequences were properly aligned and deemed as high quality were retained and concatenated into a single file. CODEML (Yang 2007) was used to calculate 4-fold degenerate sites, which are considered not to be subject to selection. Finally, MCMCtree (Yang 2007) was used to build a tree. To calibrate this tree, we used secondary calibrations from the most complete phylogenetic tree of the palm family (Baker \& Couvreur 2013). Independent substitution rates were inferred for each branch in the tree, and HKY85 (the most complex substitution model available in MCMCtree) was selected. The MCMC chain was run to gather 20,000 samples after convergence had been achieved. The first 10,000 iterations were discarded as a burn-in. Assuming a generation time of 10 years for Howea palms (Lord Howe Island Nursery, pers. comm.), we estimated the rate of substitution of the branch leading to $H$. forsteriana to be $1.3 \times 10^{-8}$ mutations per site per generation. 
Results and discussion

The demographic analyses provide strong support that Howea palms on LHI diverged in the face of ongoing gene flow. We have categorised the demographic models into four groups: (i) no population growth; (ii) population growth; (iii) no growth but heterogeneous migration across the genome; (iv) growth with heterogeneous migration across the genome. Using fastsimcoal2, where heterogeneous migration across the genome does not apply, model 5 (divergence with two periods where gene flow may vary) was most likely when no growth was modelled (Table 1). When population growth was permitted, model 5 was within 10 AIC from models 2 and 3, and therefore these three models are undistinguishable (Burnham \& Anderson 2002) (Table 1). Using $\delta a \delta i$, model 5 was always the most likely model. However, when growth and heterogeneous migration across the genome were permitted, model 5 was indistinguishable from model 3 (speciation with initial gene flow; Table 1). The more complex growth models had smaller AIC than the no growth counterparts (Table 1), pointing to a period of exponential growth following species divergence. However, for both $\delta a \delta i$ and fastsimcoal 2 the confidence intervals included the point estimates for all parameters only for the simplest scenario (i.e., without population size changes or heterogeneous rate of genome flow across the genome, Table S1). Other scenarios had greater levels of uncertainty indicating that our data were not sufficient to constrain such complex models and the point estimates for these models are likely to be unreliable. As the two demographic methods produced comparable results for the no growth scenarios, we focus our discussion on these simpler cases. In models including two distinct periods of migration, we did not constrain the earlier migration rate to be higher that the more recent migration rate. Nevertheless, in all of these models the earlier rate of gene flow is estimated to be higher than more recent rates, supporting a reduction in gene flow during speciation. A similar pattern was found during the divergence of Senecio on Mt. Etna (Filatov et al. 2016).

Figure $1 \mathrm{~b}$ shows the detailed scenario for model 5 without growth. Confidence intervals for all parameters for $\delta a \delta i$ were much wider than for fastsimcoal2 (Table S1), so we mainly discuss results from the latter. To assess the fit of the data to the model, we calculated the likelihood ratio Gstatistic (Composite likelihood ratio $=470.2$ ) and compared this to the null distribution of simulated values (Excoffier et al.2013). The observed value is in the tail of the simulated distribution (above the 99.9 percentile; range $=327.4-485.9)$. This is expected as these demographic models are a 
simplification of the real history of the species, whereas the null distribution is based on data simulated under the simple model (Excoffier et al.2013).

Migration was initially two orders of magnitude higher from the smaller $H$. forsteriana population into the larger $H$. belmoreana lineage (proportion of migrants received per generation $=4.00 \times 10^{-4}$ vs $4.7110^{-6}$; effective migrants per generation, $\mathrm{Nm}=13.01$ vs 0.27 ). This initial period was followed by a reduction in gene flow (proportion of migrants received per generation $=1.6 \times 10^{-7} \mathrm{vs}$ $3.3 \times 10^{-7} ; \mathrm{Nm}=0.01$ vs 0.02 ). This is consistent with $H$. forsteriana being derived from a belmoreana-like ancestor that colonised a new habitat in which the $H$. belmoreana genetic background was selected against. The initially high $\mathrm{Nm}($ mean $=6.64)$ is in the top $6 \%$ of values found in other examples speciation with gene flow (range = 0.00 - 25.22; Pinho \& Hey 2010; Filatov et al. 2016) and the proportion of migrants is similar to that found in the sympatric Nicaraguan cater lake cichlids $\left(7.48 \times 10^{-5}-8.51 \times 10^{-5}\right.$; Kautt et al. 2016). These migration estimates fall below those expected under population genetic definitions of sympatric speciation $(\mathrm{m}=0.5$; Fitzpatrick et al. 2008). However, it is important to note that our migration estimates are averages over long periods of time, forced by a model that has an abrupt transition from one population to two populations. If we had a model that allowed a progressive reduction in gene flow, we may have seen values close to 0.5 at the start and then a rapid reduction as the initial barriers were built. Unfortunately, we do not have the data to fit such a model. Of course, it would also have strong heterogeneity across the genome. Based on a generation time of 10 years, we found that the species initially diverged roughly 2.7 million years ago, which is older than previously estimated by phylogenetic analysis of two genes (1.92 +/- 0.52 million years ago; Savolainen et al. 2006). Allowing for different rates of migration across the genome (using $\delta a \delta i$ ) resulted in a more recent divergence time than other models at 1.41 Mya, but this fell outside the bootstrap confidence intervals. Our estimates predate the proposed mid-Pleistocene age of the current calcareous deposits of the island (Brooke 2003; Woodroffe et al. 2006; Papadopulos et al. 2013a). It is possible that colonisation was on calcareous soils pre-dating those currently on the island, and which would have eroded since then. Alternatively, colonisation of mid-Pleistocene calcareous sites may have taken place after divergence with the initial selection pressures stemming from other sources, such as water availability or salinity (Papadopulos et al. 2013b). The timing of the shift to a lower migration rate differs substantially between the methods; fastimcoal 2 points to a large reduction in migration $\sim 40,000$ years after initial divergence, whereas a $\delta a \delta i$ indicates smaller change that took place much more recently $(100,000$ year after divergence). Estimates of current population sizes $(H$. forsteriana $N_{e}=32,510 ;$ H. belmoreana $N_{e}=57,181$ ) are similar to the estimated census population size (all LHI Howea 100,000, with 2.7 times as many H. belmoreana as H. forsteriana) (Savolainen et al. 2006; Hipperson et al. 2016). The fastsimcoal2 estimated ancestral $N_{e}(424,288)$ is within the 
bounds of possibility, but is likely to be an overestimate as variation in coalescence time due to selection in the ancestral species may cause fastsimcoal2 to explain excess variance by inferring very large $N_{e}$. The $\delta a \delta i$ ancestral $N_{e}$ was an order of magnitude lower $(12,570)$, and it is therefore unclear which estimate is best. It seems likely that small initial colonising group came from a large mainland population and then grew rapidly, but this not possible to determine with our analyses. The inconsistent results for the more complex models show that our analyses are limited by the data. Refinements could be made using whole genome re-sequencing data, although the large genome size and complexity makes this challenging. Additionally, more detailed data would allow the inclusion of other parameters in more complex frameworks that have emerged recently - such as that of Roux et al (2016), which models heterogeneity in coalescence times due to selection.

Currently, reproductive isolation between the species is strong, although not complete given that occasional fertile hybrids are formed (Babik et al. 2009). Our results are consistent with the idea that initial local adaptation and post-zygotic isolation were supplemented by the rapid completion of pre-zygotic isolation through flowering time differences (Papadopulos et al. 2013b, 2014).

Furthermore, given the supporting evidence these results allow us to rule out continuous absence of gene flow, even though LHI was larger at the time of speciation (Papadopulos et al. 2011, 2013a; Linklater et al. 2018). Unlike recent genomic reanalyses of classic cases of speciation in sympatry, our results support the proposition that Howea palms must have diverged with continuous gene flow. We note, however, that genomic data by themselves may only permit the rejection of the simplest form of allopatry (Yang et al. 2017). In this sense, our demographic analyses should be seen in concert with other lines of evidence such as the geological history of LHI, lack of population structure on LHI, and the finding of candidate reproductive isolation genes (Dunning et al. 2016). Furthermore, our analyses indicate that divergence may have predated the origin of the calcarenite soils on LHI, and therefore identifying candidate 'speciation genes' with functions related to drought and salt tolerance may be more important than extremes of $\mathrm{pH}$.

\section{REFERENCES}

Al-Mssallem, I.S., Hu, S., Zhang, X., Lin, Q., Liu, W., Tan, J., et al. (2013). Genome sequence of the date palm Phoenix dactylifera L. Nat. Commun. 4: 2274. Nature Publishing Group.

Babik, W., Butlin, R.K., Baker, W.J., Papadopulos, A.S.T., Boulesteix, M., Anstett, M.C., et al. (2009). How sympatric is speciation in the Howea palms of Lord Howe Island? Mol. Ecol. 18: 3629-3638.

Baker, W.J. \& Couvreur, T.L.P. (2013). Global biogeography and diversification of palms sheds 
light on the evolution of tropical lineages. I. Historical biogeography. J. Biogeogr. 40: 274285.

Brooke, B. (2003). Quaternary calcarenite stratigraphy on Lord Howe Island, southwestern Pacific Ocean and the record of coastal carbonate deposition. Quat. Sci. Rev. 22: 859-880.

Burnham, K.P. \& Anderson, D.R. (2002). Model selection and multimodel inference: a practical information-theoretic approach. Springer, New York.

Butlin, R.K., Galindo, J. \& Grahame, J.W. (2008). Sympatric, parapatric or allopatric: the most important way to classify speciation? Philos. Trans. R. Soc. B Biol. Sci. 363: 2997-3007.

Coyne, J.A. (2011). Speciation in a small space. Proc. Natl. Acad. Sci. 108: 12975-12976.

Cruickshank, T.E. \& Hahn, M.W. (2014). Reanalysis suggests that genomic islands of speciation are due to reduced diversity, not reduced gene flow. Mol. Ecol. 23: 3133-3157.

Dunning, L.T., H. Hipperson, Baker, W.J., Butlin, R.K., Devaux, C., Hutton, I., et al. (2016). Ecological speciation in sympatric palms: 1 . Gene expression, selection and pleiotropy. $J$. Evol. Biol. 29: 1472-1487.

Edgar, R.C. (2004). MUSCLE: Multiple sequence alignment with high accuracy and high throughput. Nucleic Acids Res. 32: 1792-1797.

Excoffier, L., Dupanloup, I., Huerta-Sánchez, E., Sousa, V.C. \& Foll, M. (2013). Robust Demographic Inference from Genomic and SNP Data. PLoS Genet. 9.

Filatov, D.A., Osborne, O.G. \& Papadopulos, A.S.T. (2016). Demographic history of speciation in a Senecio altitudinal hybrid zone on Mt. Etna. Mol. Ecol. 25: 2467-2481.

Fitzpatrick, B.M., Fordyce, J.A. \& Gavrilets, S. (2008). What, if anything, is sympatric speciation? J. Evol. Biol. 21: 1452-1459.

Gavrilets, S. (2004). Fitness Landscapes and the Origin of Species. Princeton Univeristy Press, Princeton, NJ.

Gouveia-Oliveira, R., Sackett, P.W. \& Pedersen, A.G. (2007). MaxAlign: maximizing usable data in an alignment. BMC Bioinformatics 8: 312.

Grabherr, M.G., Haas, B.J., Yassour, M., Levin, J.Z., Thompson, D.A., Amit, I., et al. (2011). Fulllength transcriptome assembly from RNA-Seq data without a reference genome. Nat. Biotechnol. 29: 644-652.

Gutenkunst, R.N., Hernandez, R.D., Williamson, S.H. \& Bustamante, C.D. (2009). Inferring the joint demographic history of multiple populations from multidimensional SNP frequency data. PLoS Genet. 5.

Hipperson, H., Dunning, L.T., Baker, W.J., Butlin, R.K., Hutton, I., Papadopulos, A.S.T.T., et al. (2016). Ecological speciation in sympatric palms: 2. Pre- and post-zygotic isolation. J. Evol. Biol. 29: 2143-2156. 
Katoh, K. \& Toh, H. (2008). Recent developments in the MAFFT multiple sequence alignment program. Brief. Bioinform. 9: 286-298.

Kautt, A.F., Machado-Schiaffino, G. \& Meyer, A. (2016). Multispecies Outcomes of Sympatric Speciation after Admixture with the Source Population in Two Radiations of Nicaraguan Crater Lake Cichlids. PLoS Genet. 12: 1-33.

Lassmann, T. \& Sonnhammer, E.L.L. (2005). Kalign--an accurate and fast multiple sequence alignment algorithm. BMC Bioinformatics 6: 298.

Linklater, M., Hamylton, S.M., Brooke, B.P., Nichol, S.L., Jordan, A.R. \& Woodroffe, C.D. (2018). Development of a Seamless, High-Resolution Bathymetric Model to Compare Reef Morphology around the Subtropical Island Shelves of Lord Howe Island and Balls Pyramid, Southwest Pacific Ocean. Geosciences 8: 11.

Mallet, J., Meyer, A., Nosil, P. \& Feder, J.L. (2009). Space, sympatry and speciation. J. Evol. Biol. 22: $2332-2341$.

Martin, C.H., Cutler, J.S., Friel, J.P., Touokong, C.D., Coop, G. \& Wainwright, P.C. (2015). Complex histories of repeated gene flow in Cameroon crater lake cichlids cast doubt on one of the clearest examples of sympatric speciation. Evolution (N. Y). 69: 1406-1422.

Notredame, C., Higgins, D.G. \& Heringa, J. (2000). T-Coffee: A novel method for fast and accurate multiple sequence alignment. J. Mol. Biol. 302: 205-17.

Papadopulos, A.S.T., Baker, W.J., Crayn, D., Butlin, R.K., Kynast, R.G., Hutton, I., et al. (2011). Speciation with gene flow on Lord Howe Island. Proc. Natl. Acad. Sci. U. S. A. 108: 1318813193.

Papadopulos, A.S.T., Baker, W.J. \& Savolainen, V. (2013a). Sympatric Speciation of Island Plants: The Natural Laboratory of Lord Howe Island. In: Speciation: Natural Processes, Genetics \& Biodiversity (P. Michalak, ed). Nova Science.

Papadopulos, A.S.T., Kaye, M., Devaux, C., Hipperson, H., Lighten, J., Dunning, L.T., et al. (2014). Evaluation of genetic isolation within an island flora reveals unusually widespread local adaptation and supports sympatric speciation. Philos. Trans. R. Soc. B Biol. Sci. 369: 20130342.

Papadopulos, A.S.T., Price, Z., Devaux, C., Hipperson, H., Smadja, C.M., Hutton, I., et al. (2013b). A comparative analysis of the mechanisms underlying speciation on Lord Howe Island. $J$. Evol. Biol. 26: 733-745.

Peterson, B.K., Weber, J.N., Kay, E.H., Fisher, H.S. \& Hoekstra, H.E. (2012). Double digest RADseq: an inexpensive method for de novo SNP discovery and genotyping in model and non-model species. PLoS One 7: e37135. Public Library of Science.

Pinho, C. \& Hey, J. (2010). Divergence with Gene Flow: Models and Data. Annu. Rev. Ecol. Evol. 
Syst. 41: 215-230.

Renaut, S., Grassa, C.J., Yeaman, S., Moyers, B.T., Lai, Z., Kane, N.C., et al. (2013). Genomic islands of divergence are not affected by geography of speciation in sunflowers. Nat. Commun. 4: 1827.

Roux, C., Fraïsse, C., Romiguier, J., Anciaux, Y., Galtier, N. \& Bierne, N. (2016). Shedding Light on the Grey Zone of Speciation along a Continuum of Genomic Divergence. PLoS Biol. 14: 122.

Savolainen, V., Anstett, M.-C., Lexer, C., Hutton, I., Clarkson, J.J., Norup, M. V, et al. (2006). Sympatric speciation in palms on an oceanic island. Nature 441: 210-213.

Singh, R., Ong-Abdullah, M., Low, E.-T.L., Manaf, M.A.A., Rosli, R., Nookiah, R., et al. (2013). Oil palm genome sequence reveals divergence of interfertile species in Old and New worlds. Nature 500: 335-9. Nature Publishing Group.

Tine, M., Kuhl, H., Gagnaire, P.A., Louro, B., Desmarais, E., Martins, R.S.T., et al. (2014). European sea bass genome and its variation provide insights into adaptation to euryhalinity and speciation. Nat. Commun. 5: 5770.

Wallace, I.M., O’Sullivan, O., Higgins, D.G. \& Notredame, C. (2006). M-Coffee: combining multiple sequence alignment methods with T-Coffee. Nucleic Acids Res. 34: 1692-9.

Woodroffe, C.D., Kennedy, D.M., Brooke, B.P. \& Dickson, M.E. (2006). Geomorphological evolution of Lord Howe Island and carbonate production at the latitudinal limit to reef growth. J. Coast. Res. 22: 188-201. Coastal Education and Research Foundation.

Yang, M., He, Z. \& Shi, S. (2017). Can genomic data alone tell us whether speciation happened with gene flow? Mol. Ecol. 26: 2845-2849.

Yang, Z. (2007). PAML 4: Phylogenetic Analysis by Maximum Likelihood. Mol. Biol. Evol. 24: 1586-1591. 
Table 1. Summary statistics for model selection. The most likely models within 10 AIC are highlighted in bold.

\begin{tabular}{|c|c|c|c|c|c|}
\hline Model & Method & $\begin{array}{l}\text { Max. Ln } \\
\text { (likelihood) }\end{array}$ & $\begin{array}{l}\text { No. of } \\
\text { parameter } \\
\text { s }\end{array}$ & AIC & $\begin{array}{l}\text { Delta } \\
\text { AIC }\end{array}$ \\
\hline 1 (speciation without gene flow) & fastsimcoal2 & -175730 & 4 & 351467 & 3092 \\
\hline $\begin{array}{l}2 \text { (speciation with recent gene flow } \\
\text { following secondary contact) }\end{array}$ & fastsimcoal2 & -174992 & 7 & 349997 & 1622 \\
\hline 3 (speciation with initial gene flow) & fastsimcoal2 & -175522 & 7 & 350109 & 1734 \\
\hline $\begin{array}{l}4 \text { (speciation with constant gene } \\
\text { flow) }\end{array}$ & fastsimcoal2 & -175049 & 6 & 351059 & 2683 \\
\hline $\begin{array}{l}5 \text { (species divergence with two } \\
\text { periods where gene flow may vary) }\end{array}$ & fastsimcoal2 & -174955 & 9 & 349928 & 1552 \\
\hline $1+$ growth & fastsimcoal2 & -174898 & 6 & 349808 & 1433 \\
\hline $2+$ growth & fastsimcoal2 & -174179 & 9 & 348375 & $\mathbf{0}$ \\
\hline $3+$ growth & fastsimcoal2 & -174181 & 9 & 348377 & 2 \\
\hline $4+$ growth & fastsimcoal2 & -174265 & 8 & 348548 & 173 \\
\hline $5+$ growth & fastsimcoal2 & -174180 & 11 & 348382 & 7 \\
\hline 1 & $\delta a \delta i$ & -2602.12 & 4 & 5210 & 2705 \\
\hline 2 & $\delta a \delta i$ & -2051.04 & 7 & 4114 & 1609 \\
\hline 3 & $\delta a \delta i$ & -2055.46 & 7 & 4123 & 1617 \\
\hline 4 & $\delta a \delta i$ & -2067.86 & 6 & 4146 & 1640 \\
\hline 5 & $\delta a \delta i$ & -2012.61 & 9 & 4041 & 1536 \\
\hline $1+$ growth & $\delta a \delta i$ & -1747.95 & 6 & 3506 & 1000 \\
\hline $2+$ growth & $\delta a \delta i$ & -1384.78 & 9 & 2786 & 280 \\
\hline $3+$ growth & $\delta a \delta i$ & -1427.55 & 9 & 2871 & 366 \\
\hline $4+$ growth & $\delta a \delta i$ & -1431.38 & 8 & 2877 & 371 \\
\hline $5+$ growth & $\delta a \delta i$ & -1377.74 & 11 & 2775 & 270 \\
\hline $2+$ heterogeneous $\mathrm{M}$ & $\delta a \delta i$ & -1741.57 & 10 & 3501 & 996 \\
\hline $3+$ heterogeneous $\mathrm{M}$ & $\delta a \delta i$ & -1786.64 & 10 & 3591 & 1086 \\
\hline $4+$ heterogeneous $\mathrm{M}$ & $\delta a \delta i$ & -1785.31 & 9 & 3587 & 1081 \\
\hline 5 + heterogeneous $M$ & $\delta a \delta i$ & -1730.07 & 14 & 3486 & 981 \\
\hline $2+$ growth + heterogeneous $\mathrm{M}$ & $\delta a \delta i$ & -1255.63 & 12 & 2533 & 28 \\
\hline 3 + growth + heterogeneous M & $\delta a \delta i$ & -1241.78 & 12 & 2506 & $\mathbf{0}$ \\
\hline $4+$ growth + heterogeneous $\mathrm{M}$ & $\delta a \delta i$ & -1256.03 & 11 & 2532 & 26 \\
\hline
\end{tabular}



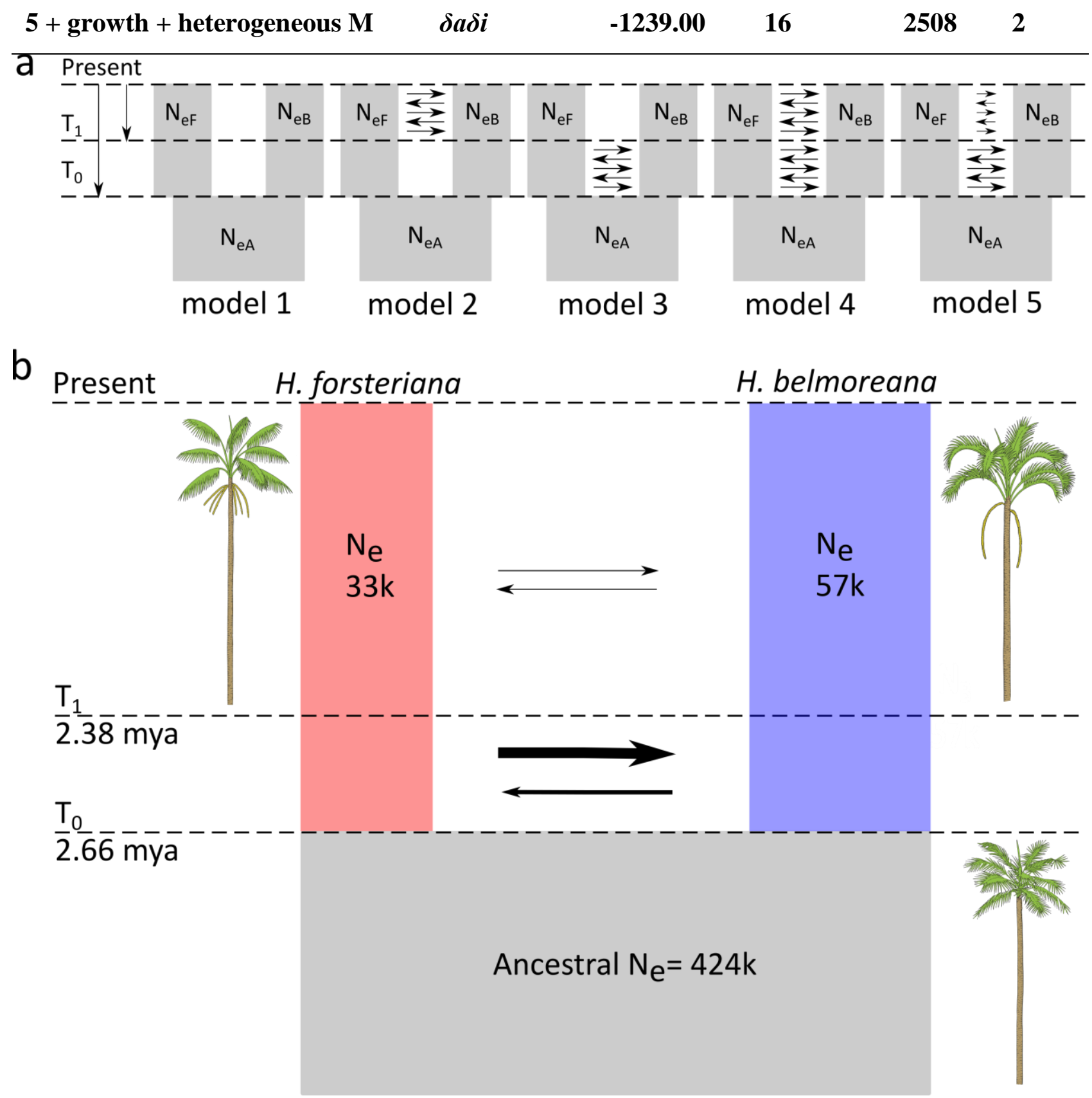

Fig. 1. Coalescence analyses of demography in Howea. (a) Five models were tested, either assuming constant population sizes, allowing for exponential population growth through time, or heterogeneous migration across the genome (18 scenarios in total). Model 1: speciation without gene flow; 2: speciation with recent gene flow following secondary contact; 3: speciation with initial gene flow; 4: speciation with constant gene flow; and 5: species divergence with two periods where gene flow may vary. (b) Parameter estimates for the best fitting model with no growth estimated in fastsimcoal2 (model 5). 
Table S1. Inferred parameters for the selected model 5

\begin{tabular}{|c|c|c|c|c|c|c|}
\hline \multirow[b]{2}{*}{ Parameter } & \multicolumn{3}{|l|}{ fastsimcoal2 } & \multicolumn{3}{|l|}{$\delta a \delta i$} \\
\hline & $\begin{array}{l}\text { Point } \\
\text { estimation }\end{array}$ & Lower CI & Upper CI & $\begin{array}{l}\text { Point } \\
\text { estimation }\end{array}$ & $\begin{array}{l}\text { Lower } \\
\text { CI }\end{array}$ & $\begin{array}{l}\text { Upper } \\
\text { CI }\end{array}$ \\
\hline & & & & & & 19889 \\
\hline Ancestral Ne & 424288 & 125435 & 547787 & 12570 & 11589 & 1 \\
\hline H. forsteriana $\mathrm{Ne}$ & 32510 & 31192 & 34006 & 31806 & 29809 & 33687 \\
\hline H. belmoreana $\mathrm{Ne}$ & 57181 & 54802 & 59951 & 49054 & 47272 & 52285 \\
\hline $\mathrm{T}_{0}$ & 2.66 & 2.56 & 4.52 & 2.71 & 0 & 40.34 \\
\hline $\mathrm{T}_{1}$ & 2.38 & 2.10 & 2.96 & 0.11 & 0 & 2.84 \\
\hline & & & $4.21 \times 10^{-}$ & & $2.97 \mathrm{x}$ & $1.45 \mathrm{x}$ \\
\hline $\mathrm{MIG}_{\mathrm{F} \rightarrow \mathrm{B}}\left(\mathrm{T}_{0}-\mathrm{T}_{1}\right)$ & $4.00 \times 10^{-4}$ & $5.87 \times 10^{-5}$ & & $5.67 \times 10^{-7}$ & $10^{-99}$ & $10^{-5}$ \\
\hline & & & $5.94 \times 10^{-}$ & & $2.01 \mathrm{x}$ & $1.27 \mathrm{x}$ \\
\hline $\mathrm{MIG}_{\mathrm{B} \rightarrow \mathrm{F}}\left(\mathrm{T}_{0}-\mathrm{T}_{1}\right)$ & $4.71 \times 10^{-6}$ & $1.32 \times 10^{-5}$ & 5 & $7.06 \times 10^{-11}$ & $10^{-138}$ & $10^{-5}$ \\
\hline $\mathrm{MIG}_{\mathrm{F} \rightarrow \mathrm{B}}(\mathrm{T} 1-$ & & & $2.27 \times 10^{-}$ & & $3.50 x$ & $6.28 x$ \\
\hline Present) & $1.57 \times 10^{-7}$ & $1.57 \times 10^{-7}$ & & $1.49 \times 10^{-7}$ & $10^{-70}$ & $10^{-6}$ \\
\hline $\begin{array}{l}\mathrm{MIG}_{\mathrm{B} \rightarrow \mathrm{F}}(\mathrm{T} 1- \\
\text { Present) }\end{array}$ & $3.30 \times 10^{-7}$ & $2.82 \times 10^{-7}$ & $\begin{array}{l}4.12 \times 10^{-} \\
7\end{array}$ & $3.78 \times 10^{-7}$ & $\begin{array}{l}5.36 \times \\
10^{-31}\end{array}$ & $\begin{array}{l}6.76 x \\
10^{-6}\end{array}$ \\
\hline
\end{tabular}

